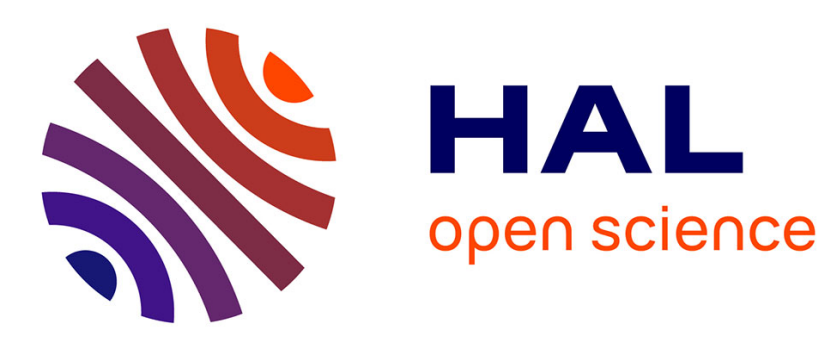

\title{
lsmear: A Variable Selection Strategy for Interval Branch and Bound Solvers
}

\author{
Ignacio Araya, Bertrand Neveu
}

\section{To cite this version:}

Ignacio Araya, Bertrand Neveu. lsmear: A Variable Selection Strategy for Interval Branch and Bound Solvers. GOW'16 XIII Global Optimization Workshop, Sep 2016, Braga, Portugal. hal-01376611

\section{HAL Id: hal-01376611 \\ https://hal-enpc.archives-ouvertes.fr/hal-01376611}

Submitted on 5 Oct 2016

HAL is a multi-disciplinary open access archive for the deposit and dissemination of scientific research documents, whether they are published or not. The documents may come from teaching and research institutions in France or abroad, or from public or private research centers.
L'archive ouverte pluridisciplinaire $\mathbf{H A L}$, est destinée au dépôt et à la diffusion de documents scientifiques de niveau recherche, publiés ou non, émanant des établissements d'enseignement et de recherche français ou étrangers, des laboratoires publics ou privés. 


\title{
lsmear: A Variable Selection Strategy for Interval Branch and Bound Solvers
}

\author{
Ignacio Araya ${ }^{1}$ and Bertrand Neveu ${ }^{2}$ \\ ${ }^{1}$ Pontificia Universidad Católica de Valparaíso, Escuela de Ingeniería Informática, Chile, ignacio.araya@pucv.cl \\ 2 Imagine LIGM Université Paris-Est, France, Bertrand.Neveu@enpc.fr
}

\begin{abstract}
Smear-based variable selection strategies are well-known and commonly used by branch-and-prune interval-based solvers. They estimates the impact of the variables on each constraint of the system by using the partial derivatives and the sizes of the variable domains. Then they aggregate these values, in some way, to estimate the impact of each variable on the whole system. The variable with the greatest impact is then selected. A problem of these strategies is that they, generally, consider all constraints equally important.

In this work, we propose a new variable selection strategy which first weights the constraints by using the optimal Lagrangian multipliers of a linearization of the original problem. Then, the impact of the variables is computed with a typical smear-based function but taking into account the weights of the constraints. The strategy is tested on classical benchmark instances outperforming significantly the classical ones.
\end{abstract}

Keywords: global optimization, branch and bound, variable selection, Lagrangian multipliers, smear function

\section{Introduction}

This paper deals with continuous global optimization (nonlinear programming) deterministically handled by interval branch and bound $(\mathrm{B} \& \mathrm{~B})$. The problem is defined by:

$\min _{x \in \boldsymbol{x}} f(x)$ s.t. $g(x) \leq 0$, where $f: \mathbb{R}^{n} \rightarrow \mathbb{R}$ is the real-valued objective (non convex) function and $g: \mathbb{R}^{n} \rightarrow \mathbb{R}^{m}$ is a vector-valued (non convex) function. ${ }^{1} x=\left(x_{1}, \ldots, x_{i}, \ldots x_{n}\right)$ is a vector of variables varying in a domain (i.e., a box) $x^{2}$. For performance and simplicity considerations, a variable $x_{o}$, with initial domain $\boldsymbol{x}_{o}=[-\infty,+\infty]$ is included in the set of variables $x$ and an additional constraint $f(x)=x_{o}$ is included in the set of constraints (actually, functions $f(x)-x_{o}$ and $x_{o}-f(x)$ are included in $g$ ). Finally, we solve an equivalent problem:

$$
\min _{x \in \boldsymbol{x}} x_{O} \text { s.t. } g(x) \leq 0
$$

Several works have been proposed for finding good branching strategies $([2,4,7,5,3])$. Smear-based methods $[4,3]$ use information on the system to obtain the variable with the greatest impact. The impact of a variable $x_{i}$ on a function $g_{j}$ is computed by means of the smear value. Consider that the current node is associated with box $\boldsymbol{x}$; the smear value is given by: smear $\left(x_{i}, g_{j}\right)=\left|\boldsymbol{J}_{j i}\right| * \operatorname{wid}\left(\boldsymbol{x}_{i}\right)$, where $\boldsymbol{J}_{j i}$ is an interval overestimate of the range of the partial derivative $\frac{\partial g_{j}}{\partial x_{i}}$ in $\boldsymbol{x} .\left|\boldsymbol{J}_{j i}\right|$ is the magnitude of the interval $\boldsymbol{J}_{j i}$, i.e., $\left|\boldsymbol{J}_{j i}\right|=\max \left(\left|\underline{J_{j i}}\right|,\left|\overline{J_{j i}}\right|\right)$.

\footnotetext{
${ }^{1}$ The branching strategies proposed in this paper can also apply to problems having equality constraints.

${ }^{2}$ An interval $\boldsymbol{x}_{i}=\left[\underline{x_{i}}, \overline{x_{i}}\right]$ defines the set of reals $x_{i}$ s.t. $\underline{x_{i}} \leq x_{i} \leq \overline{x_{i}}$. A box $\boldsymbol{x}$ is a Cartesian product of intervals $\boldsymbol{x}_{1} \times \ldots \times \boldsymbol{x}_{i} \times$ $\ldots \times \boldsymbol{x}_{n}$
} 
Selection methods based on the smear value select the variable that maximizes an aggregation of this value in the whole system.

Tawarmalani and Sahinidis [6] present an algorithm called ViolationTransfer, to estimate the impact of a variable on the problem. ViolationTransfer works with the Lagrangian function of a relaxation of the problem and an optimal solution of the relaxation $x^{\star}$. For each variable, an interval $\boldsymbol{x}_{i}^{v} \subset \boldsymbol{x}_{i}$ is defined. $\boldsymbol{x}^{v}$ is the smallest box such that it contains $x^{\star}$ and each univariate constraint $g_{j}\left(x_{1}^{\star}, \ldots, x_{i-1}^{\star}, x_{i}, x_{i+1}^{\star}, x_{n}^{\star}\right) \leq 0(j=1 . . m)$ is feasible for at least one value in $\boldsymbol{x}_{i}^{v}$.

Then, for each variable $x_{i}$, the difference between the bounds of the image of the Lagrangian function over the interval $\boldsymbol{x}_{i}^{v}$ is estimated. In each estimation all the variables are fixed except $x_{i}$. The assumption is that branching on the variable maximizing the image width is likely to improve the lower bound of the objective function in the subproblems.

In this article we propose 1smear, a new variable selection strategy for interval B\&B solvers. In a few words, the method selects the variable maximizing the smear value of the Lagrangian function of the problem. In the Lagrangian function, the Lagrange multipliers are replaced by the dual optimal of a linear approximation of the problem. Related to the ViolationTransfer strategy our approach has some important differences:

1. Ismear uses a simple linear approximation of the original problem instead of sophisticated convex relaxation techniques.

2. Ismear estimates the impact of each variable in the Lagrangian function of the original problem. The estimated impact is computed by using the smear value.

3. The computation of $\boldsymbol{x}^{v}$ requires the solver uses a reformulated problem in which multidimensional functions are replaced with either univariate or bilinear functions [6]. For the moment, and in order to maintain the simplicity and generality of the approach, lsmear uses directly $\boldsymbol{x}$ instead of $\boldsymbol{x}^{v}$.

\section{Ismear, a Smear-based strategy using optimal Lagrange multipliers}

A main issue related to the smear-based strategies is that these strategies consider all the constraints equally important. To overcome this issue we propose to estimate the impact of the constraints in the system by using the optimal Lagrange multipliers of a linear approximation of the original problem.

The lsmear method works in two phases. First, a linearization of the global optimization problem is generated. Each function $g_{j}(x)$ is approximated by using the first order term of its Taylor expansion around the midpoint of the box, i.e.,

$$
g l_{j}(x)=g_{j}(\operatorname{mid}(\boldsymbol{x}))+\sum_{i=1}^{n} \operatorname{mid}\left(\boldsymbol{J}_{j i}\right) \cdot\left(x_{i}-\operatorname{mid}\left(\boldsymbol{x}_{i}\right)\right)
$$

where $\boldsymbol{J}_{j i}$ is an interval overestimation of the image of $\frac{\partial g_{j}}{\partial x_{i}}$ over $\boldsymbol{x}$. Note that instead of using the partial derivatives in the midpoint of the box we use the midpoint of the overestimation of the partial derivatives (i.e., $\operatorname{mid}\left(\boldsymbol{J}_{j i}\right)$ ).

The generated linear optimization problem includes the bound constraints, i.e., $\underline{x_{i}} \leq \boldsymbol{x}_{i} \leq$ $\overline{x_{i}}$, and it is solved by using the simplex method ${ }^{3}$. If an optimum exists, then a second phase is carried out. In this phase, the strategy computes the smear value of the following function:

$$
L(x)=x_{o}+\sum_{i=1}^{m} \lambda_{j}^{*} g_{j}(x)
$$

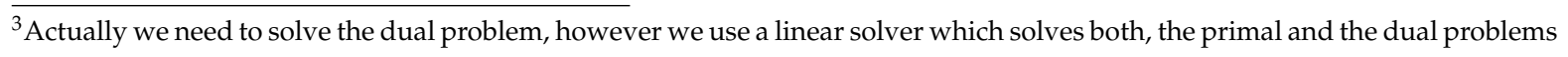


where $\lambda^{*}$ corresponds to the dual optimal solution of the linear problem. The function $L$ is equivalent to the Lagrangian of (1) but the Lagrange multipliers have been replaced by the optimal Lagrange multipliers of the linear approximation. Thus, the problem of minimizing $L(x)$ can be seen as a rough approximation of the original optimization problem. The interesting thing about $L$ is that it aggregates the objective function and the constraints of the problem in only one function, thus, computing the smear value of each variable in $L$ offers an estimation of the impact of the variable in the original problem. We believe that this estimation is fairer than the one computed by the classical smear-based strategies because each constraint is, in a certain way, being pondered according to its influence on the optimal value. Finally, same as the other Smear-based strategies, the variable with the greater impact is selected for bisection.

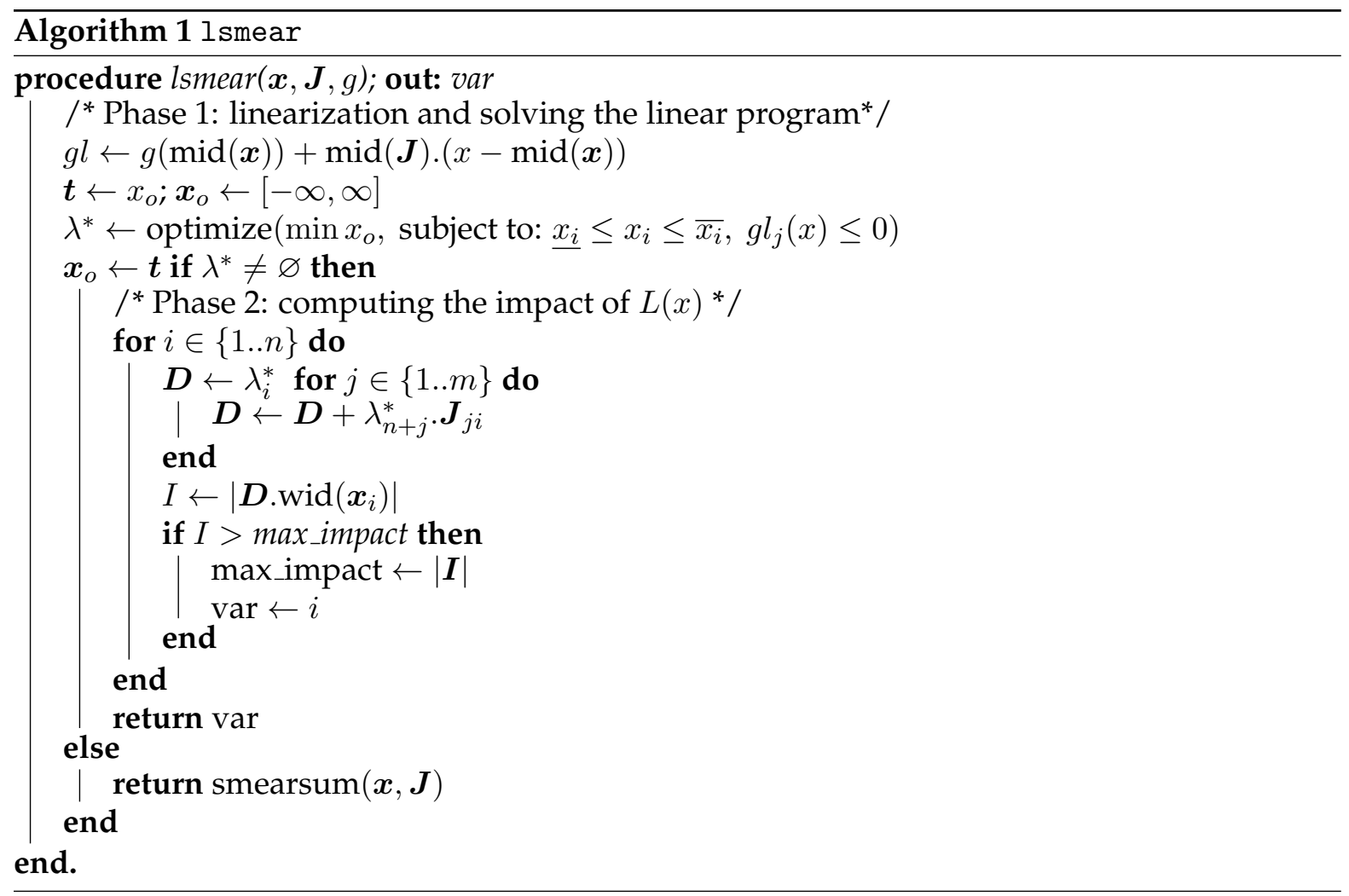

Algorithm 1 shows our approach. $\boldsymbol{J}$ corresponds to the Jacobian matrix which contains the interval overestimations of the partial derivatives over $\boldsymbol{x}$. In the linear program (Phase 1), the interval related to the objective variable is unbounded to enhance the chances for successful finding an optimal solution. In Phase 2 , for each variable $x_{i}$ we first compute $\boldsymbol{D}$, which is an interval overestimation of $\frac{\partial L}{\partial x_{i}}$ over $\boldsymbol{x}$. The overestimation is obtained by adding the products of the interval partial derivatives on each constraint $\left(\boldsymbol{J}_{j i}\right)$ and the corresponding dual optimal value $\left(\lambda_{n+j}^{*}\right) . D$ is initialized with the dual optimal value related to the bounded constraint, i.e., $\lambda_{i}^{*}$ (the partial derivative related to the $i$-th bound constraint over the variable $x_{i}$ is 1 ). Then, the smear impact of the variable is computed as the magnitude of the product of the interval partial derivative and the width of the related interval. The variable with the maximum impact is saved and returned. If the linear program does not have solutions or if the optimal value is unbounded, then the smearsum method is launched instead. This method uses the same Jacobian matrix received by the lsmear one. 


\section{Experiments}

In order to validate our approach, we implemented lsmear in IbexOpt, a state-of-the-art optimizer of the Interval-Based EXplorer library (Ibex ([1])). All the experiments were run on a server PowerEdge T420, with 2 quad-processors Intel Xeon, $2.20 \mathrm{GHz}$ and 8 GB RAM.

The instances were selected from the series 1 and 2 of the COCONUT constrained global optimization benchmarks. ${ }^{4}$ We selected all the problems solved by some strategy in a time comprised between $2 \mathrm{~s}$ and 3600 s (66 instances). Each strategy was run 5 times on each instance and the average CPU time was reported.

We compared the proposed strategy lsmear with some of the classical variable selection strategies: round-robin (rr), largest-first (lf), smearsum (ssum), smearmax (smax) and smearsumrel (ssr). Figure 1 summarizes the comparison among the six strategies.

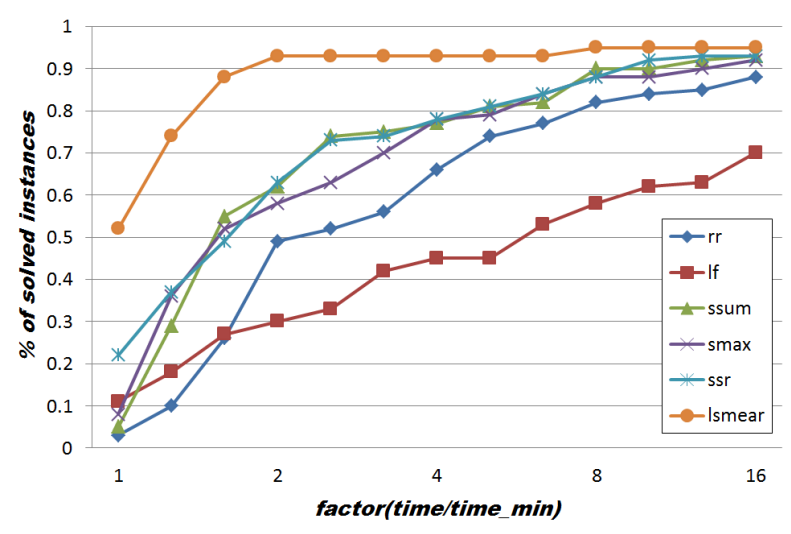

Figure 1. Performance profile.

Each curve reports the percentage of instances solved by the corresponding strategy in less than factor times the best reported CPU time. From the results we observe that 1smear clearly outperforms all the classical variable selection strategies. Also note that more than $90 \%$ of the instances are solved by lsmear in less than twice the best CPU time reported by all the strategies.

\section{References}

[1] G. Chabert and L. Jaulin. Contractor Programming. Artificial Intelligence, 173:1079-1100, 2009.

[2] Tibor Csendes and Dietmar Ratz. Subdivision direction selection in interval methods for global optimization. SIAM Journal on Numerical Analysis, 34(3):922-938, 1997.

[3] Eldon Hansen and G William Walster. Global optimization using interval analysis: revised and expanded, volume 264. CRC Press, 2003.

[4] R Baker Kearfott and Manuel Novoa III. Algorithm 681: INTBIS, a portable interval Newton/bisection package. ACM Transactions on Mathematical Software (TOMS), 16(2):152-157, 1990.

[5] R Moore. Interval analysis, vol. 60, 1966.

[6] Mohit Tawarmalani and Nikolaos V Sahinidis. Global optimization of mixed-integer nonlinear programs: A theoretical and computational study. Mathematical programming, 99(3):563-591, 2004.

[7] G. Trombettoni, I. Araya, B. Neveu, and G. Chabert. Inner regions and interval linearizations for global optimization. In $A A A I$, pages 99-104, 2011.

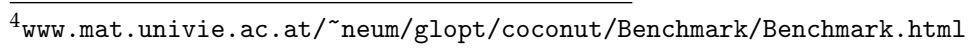

